\title{
Single Axis Geometry by Fitting Conics
}

\author{
Guang Jiang ${ }^{1,4}$, Hung-tat Tsui ${ }^{1}$, Long Quan ${ }^{2}$, and Andrew Zisserman ${ }^{3}$ \\ 1 Dept. of Electronic Engineering, The Chinese University of Hong Kong, \\ New Territory, Hong Kong \\ \{gjiang,httsui\}@ee.cuhk.edu.hk
}

2 Dept. of Computer Science, Hong Kong University of Science and Technology, Kowloon, Hong Kong quan@cs.ust.hk

3 Department of Engineering Science, University of Oxford, Parks Road, Oxford OX1 3PJ, UK

az@robots.ox.ac.uk

4 School of Technical Physics, Xidian University, Xi'an 710071, P.R. China

\begin{abstract}
In this paper, we describe a new approach for recovering 3D geometry from an uncalibrated image sequence of a single axis (turntable) motion. Unlike previous methods, the computation of multiple views encoded by the fundamental matrix or trifocal tensor is not required. Instead, the new approach is based on fitting a conic locus to corresponding image points over multiple views. It is then shown that the geometry of single axis motion can be recovered given at least two such conics. In the case of two conics the reconstruction may have a two fold ambiguity, but this ambiguity is removed if three conics are used. The approach enables the geometry of the single axis motion (the 3D rotation axis and Euclidean geometry in planes perpendicular to this axis) to be estimated using the minimal number of parameters. It is demonstrated that a Maximum Likelihood Estimation results in measurements that are as good as or superior to those obtained by previous methods, and with a far simpler algorithm. Examples are given on various real sequences, which show the accuracy and robustness of the new algorithm.
\end{abstract}

\section{Introduction}

Acquiring 3D models from single axis motion sequences, particularly turntables, has been widely used by computer vision and graphics researchers. The key component of the 3D reconstruction is the recovery of the rotation angles. Traditionally, rotation angles are obtained by careful calibration $[4,17,22,23]$. Fitzgibbon et al. [8] extended the single axis approach to recover unknown rotation angles using a projective geometry model of the motion. In their method, corresponding points are carefully tracked over each pair of images for the fundamental matrices and each triplet of images for the trifocal tensors. Mendonça et al. [15] recover the rotation angles from profiles of surfaces. The search for corresponding points is transformed into a search for epipolar tangencies. All of the above papers have some discussion on the invariants in this special single 
axis motion. However the ideal points $\mathbf{i}$ and $\mathbf{j}$, which play a very important role in rotation angle recovery, are not discussed explicitly.

Recently a new method has been proposed [11] which does not incur the expense of computing trifocal tensors or of the nonlinear optimization involved in computing epipolar tangencies. Instead, corresponding points in different images are fitted to a conic. It is then shown that rotation angles can be directly computed from only one conic and one fundamental matrix. The remaining geometric quantities can be obtained using more conics.

In this paper, we show further that even one fundamental matrix is unnecessary. All single axis geometry can be computed from at least two conics by directly calculating the image of the circular points. Thence all invariant quantities of the single axis motion can be computed. The advantage of the new method over the existing ones is straightforward. First, it is intrinsically a multiple view approach as all geometric information from the whole sequence is nicely summarized in the conics! This contrasts with the computation of fundamental matrices and trifocal tensors which use only a subsequence of 2 and 3 views respectively. Second, as will be shown in section 4 , the essential geometry of the image single axis geometry may be specified by six parameters and this may be minimally estimated from two conics (a total of 10 parameters). Previous methods have involved estimating more than this minimum number of parameters, e.g. 18 tensor parameters from 3 views.

The paper is organized as follows. A review of the geometry of single axis motion is given in Section 2. Section 3 describes the invariants under the single axis motion case and the rotation angle recovery based on these invariants. A Maximum Likelihood Estimation method for these invariants and conics fitting is given in Section 4. Section 5 demonstrates two experiments. One is on the reconstruction of the Hannover dinosaur from a turntable image sequence. The other is on a reconstruction of a girl who is seated in a rotating chair. The latter is a short video sequence and we want to show that 3D photography can be easily done. Finally, a short conclusion is given in Section 6 .

\section{Review of the Geometry of Single Axis Motion}

Throughout the paper, scalars are denoted in plain letters or lower case Greek. Assuming a pin-hole camera model, the object space may be considered as embedded in $\mathcal{P}^{3}$ and the image space embedded in $\mathcal{P}^{2}$. Vectors in $\mathcal{P}^{2}$ are denoted in lower case boldface and vectors in $\mathcal{P}^{3}$ and matrices in upper case boldface. The camera performs the projection from $\mathcal{P}^{3}$ to $\mathcal{P}^{2}$, and can be represented by a $3 \times 4$ matrix $\mathbf{P}_{3 \times 4}$ of rank 3 whose kernel is the projection center. The relationship between a point $\mathbf{X}$ in $\mathcal{P}^{3}$ and a point $\mathbf{x}$ in $\mathcal{P}^{2}$ can be written as

$$
\lambda \mathbf{x}=\mathbf{P}_{3 \times 4} \mathbf{X},
$$

where $\mathbf{x}$ and $\mathbf{X}$ are in homogeneous coordinates and $\lambda$ is a non-zero scalar.

A typical set up for single axis motion consists of a stationary CCD camera in front of a turntable on which the object to be reconstructed is placed $[17,18]$. The internal parameters of the camera are assumed fixed. 


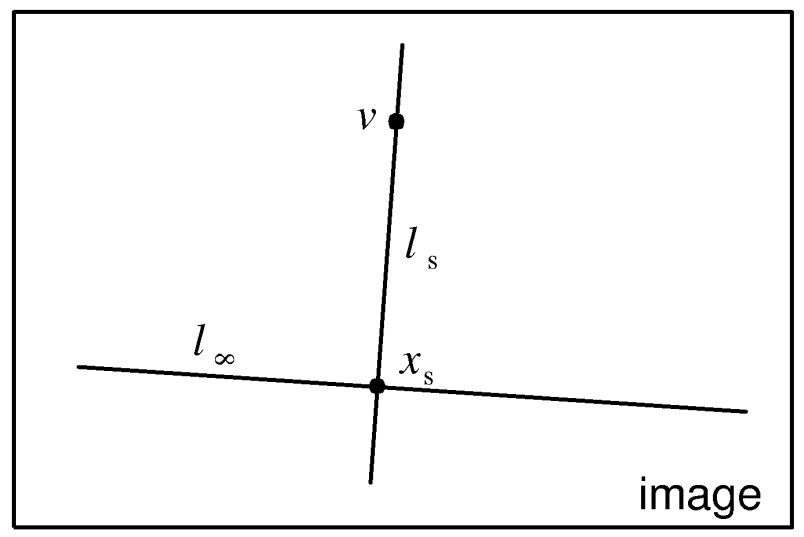

Fig. 1. Fixed image entities

For a single axis motion, without loss of generality, the rotation axis of the turntable is chosen to be the $z$-axis the world coordinate. Then each point on the object is moving in its plane that is perpendicular to the $z$-axis. Indeed, there is a pencil of horizontal planes. The invariants related to the geometry of single axis motion have been established in [8]. These invariants on the image plane are:

1. The line $\mathbf{l}_{s}$ which is the image of the rotation axis.

2. The line $\mathbf{l}_{\infty}$ which is the image of the intersection line for all horizontal planes. Actually, it is the vanishing line of all the horizontal planes.

3. The point $\mathbf{x}_{s}$ which is the image of the intersection of the rotation axis with the horizontal plane through the camera center.

4. The point $\mathbf{v}$ which is the vanishing point of the rotation axis.

These fixed image entities are illustrated schematically in Figure 1.

\section{Single Axis Geometry and Conic Loci}

Our first remark is that the trajectory of the corresponding points in different images of any given space point, displayed in any particular image plane, is a conic by the very definition of single axis motion (Figure 2) [19]. This extremely
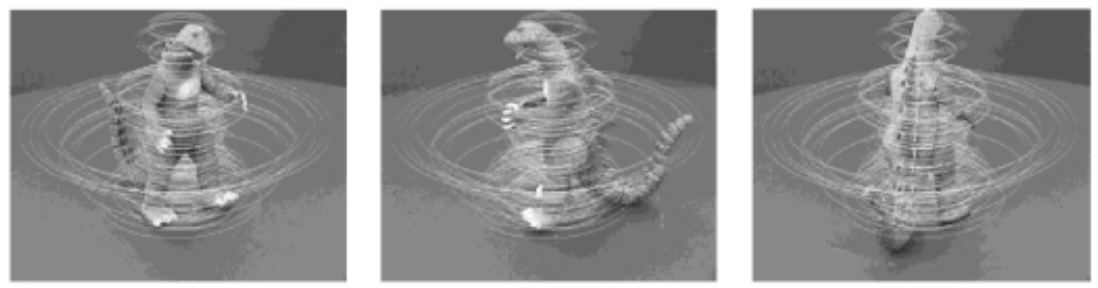

Fig. 2. Dinosaur sequence and Conics 
trivial observation that the previous methods fail to exploit is the starting point of our new methods of determining single axis geometry from conic loci. It will be seen that we can compute the geometry by simply fitting conics to the tracked points over at least five images. A minimum of 2 conics is necessary to recover the entire geometry of single axis motion.

\subsection{Computing the Image of the Circular Points from Conics}

The first essential observation is that all conic locus of image points of an object from a single axis motion contain the image of the circular points.

This can be easily proved by the fact that the trajectory of a given object point in space from a single axis motion is always a circle and different points therefore give circles of different radius on different planes which are all parallel and perpendicular to the single rotation axis. All circles go through their respective circular points of the plane they are lying on by the very definition of the circular points [20]. As all these supporting planes are parallel, i.e. they share a common line at infinity on which the circular points lie. We may thus conclude that all circles of different radius on different parallel planes from the single axis motion share the common pair of circular points $\mathbf{i}$ and $\mathbf{j}$. Because this is a projective property it remains true for the projection onto any image plane. In any particular image plane, this means that all conic loci of corresponding points intersect in the pair of common circular points $\mathbf{i}$ and $\mathbf{j}$. Thus, by just computing intersection points of at least two conic loci, we obtain the image of the circular points, and also the vanishing line of the parallel planes.

Consider the intersection of a pair of conics, there are always 4 intersection points including complex and infinite points according to Bezout's theorem. In terms of real intersection points, they may be $0,1,2,3$, or 4 according to the configurations.

- For the case of 1 or 2 real intersection points, it is straightforward that the only pair of complex conjugate points is the image of the circular points.

- It is generally impossible to have 3 or 4 real intersection points if the conics are real perspective image of the circles from the single axis motion by its definition.

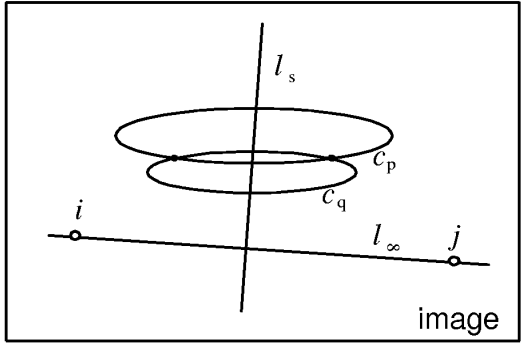

(a)

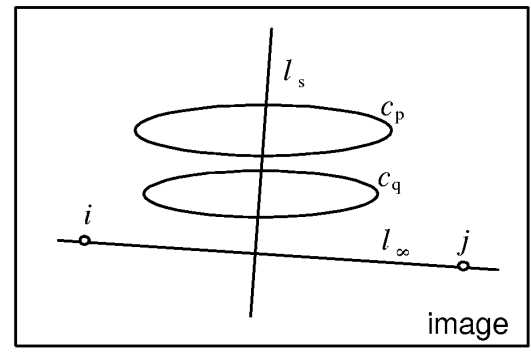

(b)

Fig. 3. The intersections of two conics 
- The most difficult case is when there is no real intersection points. This is in fact very common if we just look at the illustrative conic loci in Figure 3. In this case, we obtain two pairs of complex conjugate points. There is a reconstruction ambiguity coming from the ambiguity of the two pairs of complex conjugate points. Of course, this ambiguity can be immediately removed as soon as we have more than 2 conics.

\subsection{The Complete Computation Method}

After having described the key components of computing the image of the circular points from at least two conics, we can summarize the complete method of computing rotational angles and the underlying single axis geometry.

1. Fit two conics $\mathbf{C}_{p}$ and $\mathbf{C}_{q}$ to all tracked points over the sequence for two given points respectively. At this stage, two points tracked over at least 5 images are needed.

2. Compute the intersection points of the two conics $\mathbf{C}_{p}$ and $\mathbf{C}_{q}$. We may have either one pair of complex conjugates or two pairs of complex conjugates. If there is a unique pair of complex conjugates, the image of circular points $\mathbf{i}$ and $\mathbf{j}$ are exactly this pair of complex conjugates. However if there are two possible pairs of complex conjugate points, we obtain two possible solutions. This double solution ambiguity can be removed by using any additional conic.

3. Compute the vanishing line

$$
\mathbf{l}_{\infty}=\mathbf{i} \times \mathbf{j}
$$

4. Obtain the projection of the two circle centers as the pole of the line $\mathbf{l}_{\infty}$

$$
\begin{aligned}
& \mathbf{o}_{p}=\mathbf{C}_{p}^{-1} \mathbf{l}_{\infty}, \\
& \mathbf{o}_{q}=\mathbf{C}_{q}^{-1} \mathbf{l}_{\infty} .
\end{aligned}
$$

5. Compute the rotation axis as

$$
\mathbf{l}_{s}=\mathbf{o}_{p} \times \mathbf{o}_{q} .
$$

6. Compute the angular motion between two views from the tracked points. i.e. points $\mathbf{a}$ and $\mathbf{b}$ on two views are tracked points on conic $\mathbf{C}_{p}$, the rotation angle of the two views can be calculated by using Laguerre's formula [20]

$$
\theta_{a b}=\frac{1}{2 i} \log \left(\left\{\mathbf{l}_{o_{p} a}, \mathbf{l}_{o_{p} b} ; \mathbf{l}_{o_{p}}, \mathbf{l}_{o_{p} j}\right\}\right) .
$$

The fixed image entities for the single axis motion deduced from two conics are shown in Figure 4.a and the rotation angle with respected to the image entities is shown in Figure 4.b.

Compare with Figure 1, the vanishing point $\mathbf{v}$ of the rotation axis cannot be obtained from the conics. This means that although metric structure in all planes perpendicular to the rotation axis can be accurately determined, the reconstruction along the rotation axis has a $1 \mathrm{D}$ projective ambiguity. This same result is also shown by Fitzgibbon et al. [8] and other researchers $[1,16,24]$ which have two parameters not determined. The ambiguity can be removed by specifying camera aspect ratio and parallel vertical scene lines. 


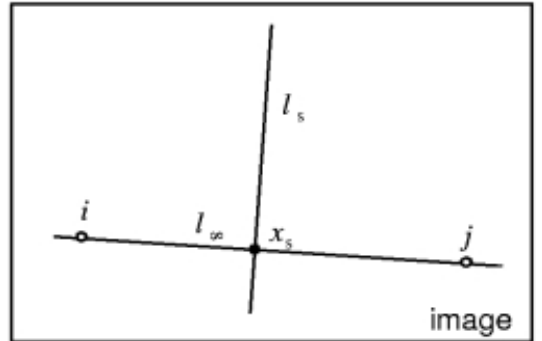

(a)

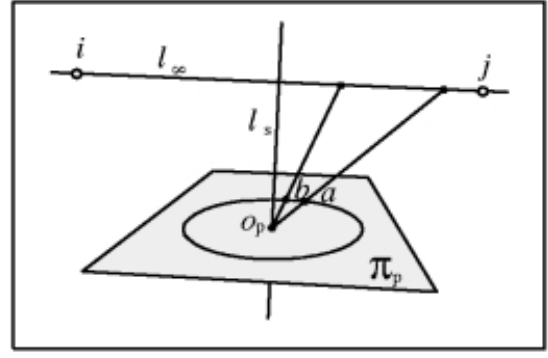

(b)

Fig. 4. (a) Fixed image entities deduced from conics. (b) The rotation angle with respect to the image entities.

\subsection{Recovering Lost Angles}

In practice, some points are missing in some views along the tracking path. The rotation angles for these views in which the tracked points are missing cannot be recovered. We show in this section how to find the 'missing' corresponding points and then how to recover the angles of these views.

Figure 5 illustrates this configuration to help the following development. The conic $\mathbf{C}$ on the plane $\pi_{1}$ has been obtained from at least a point tracked over five images. The point $\mathbf{a}$ is visible in views $m_{1}$ and $m_{2}$ as $\mathbf{a}_{1}$ and $\mathbf{a}_{2}$, but missing in view $m_{3}$, so the point $\mathbf{a}_{3}$ is not available. However, a point $\mathbf{b}$ is available in the three views $m_{1}, m_{2}$, and $m_{3}$ as $\mathbf{b}_{1}, \mathbf{b}_{2}$ and $\mathbf{b}_{3}$. Let us assume its unknown conic trajectory is the conic $\mathbf{C}_{i}$ on the plane $\pi_{i}$. As we know the two planes $\pi_{1}$ and $\pi_{i}$ are parallel in space, they therefore share the same vanishing line $\mathbf{l}_{\infty}$ and the circular points $\mathbf{i}$ and $\mathbf{j}$. Since the rotation angle between the view $m_{1}$ and $m_{2}$ is known from the conic $\mathbf{C}$. We may use Laguerre's formula for the point $\mathbf{b}$ on the plane $\pi_{i}$ :

$$
\theta_{12}=\frac{1}{2 i} \log \left(\left\{\mathbf{l}_{o_{1} b_{1}}, \mathbf{l}_{o_{1} b_{2}} ; \mathbf{l}_{o_{1} i}, \mathbf{l}_{o_{1} j}\right\}\right),
$$

where the point $\mathbf{o}_{1}$ is the only unknown vector of the image of the circle center. Since $\mathbf{o}_{1}$ lies on $\mathbf{l}_{s}$, the one unknown component of this point can be calculated from the known cross ratio $\left\{\mathbf{l}_{o_{1} b_{1}}, \mathbf{l}_{o_{1} b_{2}} ; \mathbf{l}_{o_{1} i}, \mathbf{l}_{o_{1} j}\right\}$.

From the reconstructed point $\mathbf{o}_{1}$, the rotation angle $\theta_{23}$ between the view $m_{2}$ and $m_{3}$ can be obtained.

$$
\theta_{23}=\frac{1}{2 i} \log \left(\left\{\mathbf{l}_{o_{1} b_{2}}, \mathbf{l}_{o_{1} b_{3}} ; \mathbf{l}_{o_{1} i}, \mathbf{l}_{o_{1} j}\right\}\right) .
$$

Once the angle $\theta_{23}$ has been determined, Laguerre's formula can be applied again to the computation of the line $\mathbf{l}_{o a_{3}}$. By intersecting the line $\mathbf{l}_{o a_{3}}$ with the conic $\mathbf{C}$, the missing point $\mathbf{a}_{3}$ is recovered. 


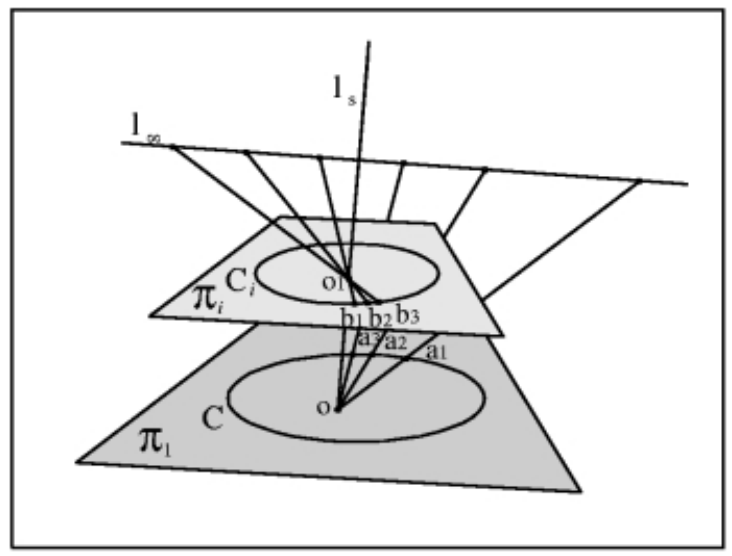

Fig. 5. Finding missing points and recovering missing angles.

\section{Invariants Estimation and Conic Fitting Based on Maximum Likelihood Estimation}

It has been shown that the geometry of single axis motion can be recovered given at least two conics. With the minimum of two conics the reconstruction may have a two fold ambiguity, but one or more additional conics makes the solution unique. In this section, a Maximum Likelihood Estimation (MLE) method is given for simultaneous estimation of the fixed geometric entities and conics.

As mentioned earlier, each point on the object is moving on its plane which is perpendicular to the $z$-axis and different points therefore form a pencil of horizontal planes. For each such plane, there is a plane homography $\mathbf{H}$ which maps the conic in the image plane to the circle lying on the horizontal plane,

$$
\mathbf{C}_{\text {cirle }}=\mathbf{H}^{-\top} \mathbf{C}_{\text {conic }} \mathbf{H}^{-1} \text {. }
$$

And we assume the center of the circle is the origin of the plane and its radius is one. We will derive a formula for this homography which will be used to parametrize the MLE.

The homography can be decomposed into a concatenation of five matrices $\mathbf{R}, \mathbf{S}, \mathbf{T}, \mathbf{A}$ and $\mathbf{P}_{u}$, representing rotation, isotropic scaling, translation, affine and 'pure projective' transformations respectively $[12,6,13]$ :

$$
\mathbf{H}=\operatorname{RSTAP}_{u}
$$

where

$$
\mathbf{P}_{u}=\left[\begin{array}{lll}
1 & 0 & 0 \\
0 & 1 & 0 \\
l_{1} & l_{2} & l_{3}
\end{array}\right]
$$


can be determined by the vanishing line $\mathbf{l}_{\infty}=\left(l_{1}, l_{2}, l_{3}\right)^{\top}$.

$$
\mathbf{A}=\left[\begin{array}{ccc}
\frac{1}{\beta} & -\frac{\alpha}{\beta} & 0 \\
0 & 1 & 0 \\
0 & 0 & 1
\end{array}\right]
$$

$(\alpha \mp i \beta, 1,0)^{T}$ is the circular points on the affine plane which can be obtained by $\mathbf{P}_{u}$ through circular points $\mathbf{i}$ and $\mathbf{j}$. It is clear that the degree of freedom of the circular points is four. Two for determining the 'pure projective' matrix and two for determining the affine matrix. As shown in Figure 4, the other fixed image entity is the rotation axis $\mathbf{l}_{s}$, which has two d.o.f.

$$
\mathbf{T}=\left[\begin{array}{ccc}
1 & 0 & -t_{1} \\
0 & 1 & -t_{2} \\
0 & 0 & 1
\end{array}\right],
$$

$\left(t_{1}, t_{2}, 1\right)^{\top}$ is the pole of vanishing line $\mathbf{l}_{\infty}$ with respect to conic $\mathbf{C}_{n}$ on the metric plane. Since the pole is constrained by the rotation axis $\mathbf{l}_{s}$, only one degree of freedom exists in the translation matrix.

$$
\mathbf{S}=\left[\begin{array}{lll}
s & 0 & 0 \\
0 & s & 0 \\
0 & 0 & 1
\end{array}\right],
$$

After processing by the matrices of $\mathbf{P}_{u}, \mathbf{A}$ and $\mathbf{T}$, the conic is transformed into a circle with center at the original point. The isotropic scaling matrix scales the circle to a circle with radius unity. There is one d.o.f. for each conic. Since the rotation matrix does not affect the circle with center at the original point, no degree of freedom lies in the matrix $\mathbf{R}$ for the circle.

In summary, there are in total 6 d.o.f. for the fixed entities $(2$ for each of the two circular point, 2 for $\mathbf{l}_{s}$ ) and 2 for each conic (which correspond to where the center is along the rotation axis (1) and its radius (1)). The cost function for the MLE involves minimizing the sum of squared geometric distances (one for each of the $m$ measured points) over all $6+2 n$ parameters for the fixed entities and $n$ conics.

$$
\mathcal{C}=\sum_{n} \sum_{m} d_{\perp}(\mathbf{x}, \mathbf{C})^{2} .
$$

However, as is shown in [10] this nonlinear cost function $\mathcal{C}$ can be approximated as

$$
d_{\perp}(\mathbf{x}, \mathbf{C})^{2}=\frac{\left(\mathbf{x}^{T} \mathbf{C x}\right)^{2}}{4\left((\mathbf{C x})_{1}^{2}+(\mathbf{C x})_{2}^{2}\right)},
$$

where $(\mathbf{C x})_{i}$ denotes the $i$-th component of the 3 -vector $\mathbf{C x}$. This function is optimized using the standard Levenberg-Marquart algorithm.

The $6+2 n$ parameters are initialized by the method in Section 3 as follows. First, each conic is fitted to corresponding points from more than 4 views $[3,7]$. Then the pole of each conic with respect to the vanishing line is calculated, and the point on the rotation axis which is nearest to the pole is used to estimate 
the initial value of $t_{1}$ ( 1 dof). Now, each conic can be transformed into a circle. The radius of this circle determines the initial value of $s$ ( 1 dof). Finally, each conic is mapped to a unit circle with center at the origin and the points on the conic is mapped to the points near the unit circle for the optimum procedure.

\section{Experiments}

The new algorithm of computing single axis geometry from only conics has been implemented. The whole computational procedure is simple and robust. We just give two examples to demonstrate the method. The first example is from the popular dinosaur image sequence from the University of Hannover. In this experiment, we have introduced a robust method for computing the rotation angles. We also show directly the projective meaning of rotation angles in an image plane. Another example is a video sequence of a girl in a rotating chair. This example also shows the practicality of this algorithm and the ease of computation.

The dinosaur sequence contains 36 views from a turntable with a constant 10 degrees angular motion. The angular accuracy is about 0.05 degrees[17]. Figure 6.a shows the tracking of some interest points of the sequence. Results of conic fitting of the same sequence using MLE is shown in Figure 6.b. Figure 7 shows the results of recovered rotation angles.

Actually, the rotation angles can be labeled on the vanishing line after randomly selecting a reference point. For instance, we may select the cross point of lines $\mathbf{l}_{\infty}$ and $\mathbf{l}_{s}$ in Figure 8 as the reference point for zero rotation angle. Any rotation angle can then be computed directly by applying Laguerre's formula and labeled on the vanishing line.

For any fitted conic, the intersections on the vanishing line with the lines joining the conic pole and known corresponding points represent the angles of

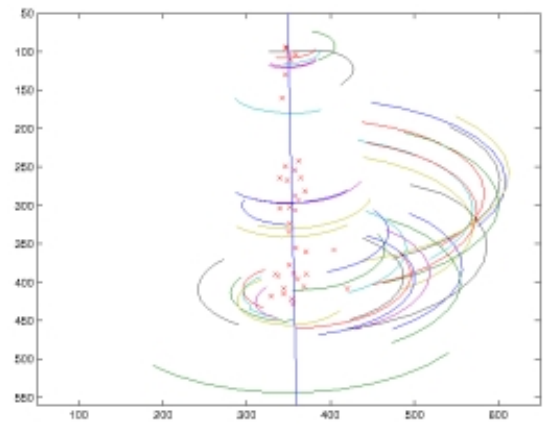

(a)

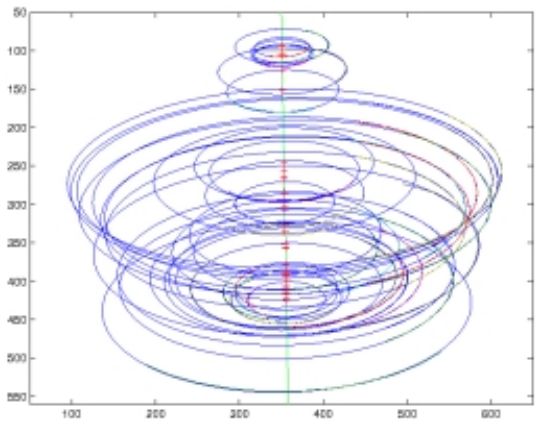

(b)

Fig. 6. (a) Some tracked points from the dinosaur sequence. The marks of ' $x$ ' indicate the initial poles of the direct fitted conics with respected to the vanishing line. (b) Fitted conics with the MLE. The marks of ' + ' indicate the poles of the MLE fitted conics with respected to the vanishing line. The marks ' + ' are neatly located on the rotation axis. 


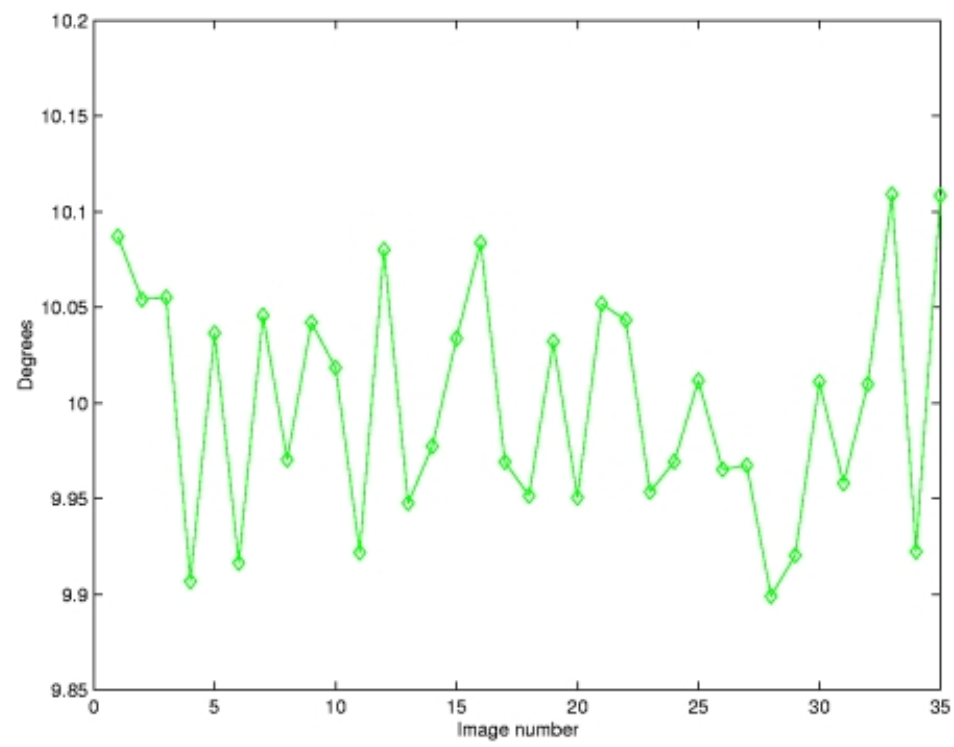

Fig. 7. Recovered rotation angles for the whole sequence of 36 views.

views with respect to the reference point. Thus, the angles (with respect to the reference point) of unknown corresponding points can be marked out on the vanishing line with the recovered rotation angles. Then, an unknown corresponding point on the conic is given by the intersection between the conic and the line joining its angle mark on the vanishing line and the conic pole. The corresponding points in all views (Figure 9) can be determined.

Using the Shape-from-Silhouettes approach described in [5,17], the horizontal slices of the dinosaur model can be obtained as illustrated in Figure 10. As the vanishing point $\mathbf{v}$ is unknown, there remains unknown ratios among slices and the reconstruction is up to two projective parameters as shown in Figure 11.

With a reasonable choice of the ratio, full reconstruction is obtained as illustrated in Figure 12.

The second example is of a girl in a rotating chair. The reconstruction is done from a short video sequence. Figure 13 shows three images captured from the video. Vertical lines in the background (Figure 13.c) are used for locating the vanishing point $\mathbf{v}$ along the rotation axis. We also assume the camera aspect ratio is one. Figure 14 shows the reconstructed 3D points in different views. Figure 15 shows the reconstructed girl.

\section{Conclusion}

We have presented a novel intuitive method of computing single axis geometry by fitting conic loci only to the corresponding points. The novelty of this algorithm is that it does not need to calculate any multiple view geometry such as 


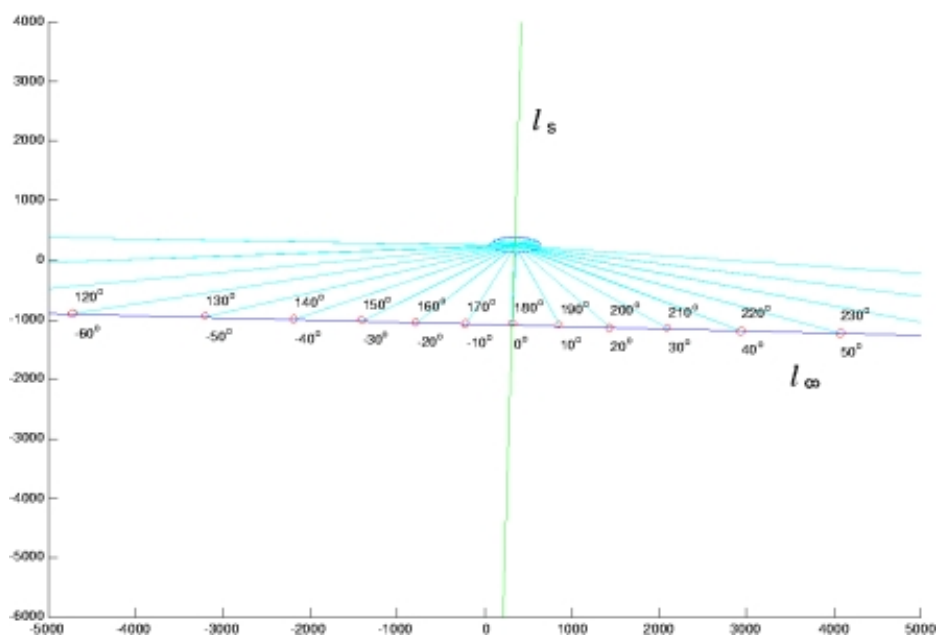

Fig. 8. Labeled rotation angles on the vanishing line (Dinosaur sequence). By selecting one reference point on the vanishing line, any rotation angle can be labeled on the vanishing line according to the Laguerre's formula.

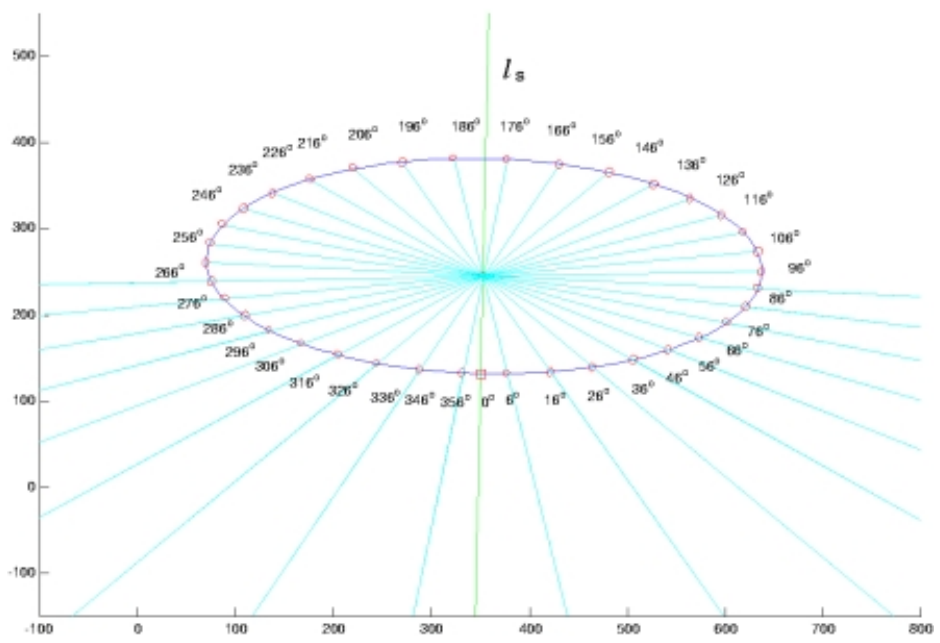

Fig. 9. Recovered corresponding points on one conic (Dinosaur sequence). After knowing the rotation angles in 36 views, with the fitted conic and a point on the conic, the corresponding points in 36 views can be recovered.

fundamental matrices and trifocal tensors. We need only to compute two, or at most three conics without any other geometric quantities in our new approach. Using MLE and more than three conics can improve the estimation and reconstruction. The algorithm is simple and robust and the number of parameters to be estimated is substantially reduced compared to previous approaches. This fact is verified by experiments on real data showing very good results. 


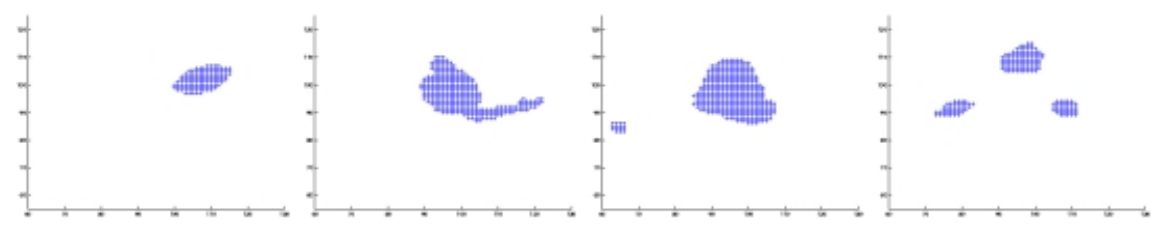

Fig. 10. Four Slices of the dinosaur.
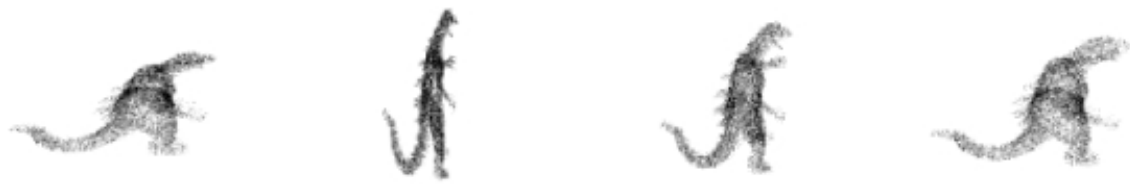

Fig. 11. 3D reconstruction up to two projective parameters. Four reconstructions are displayed with different choices of unknown parameters.
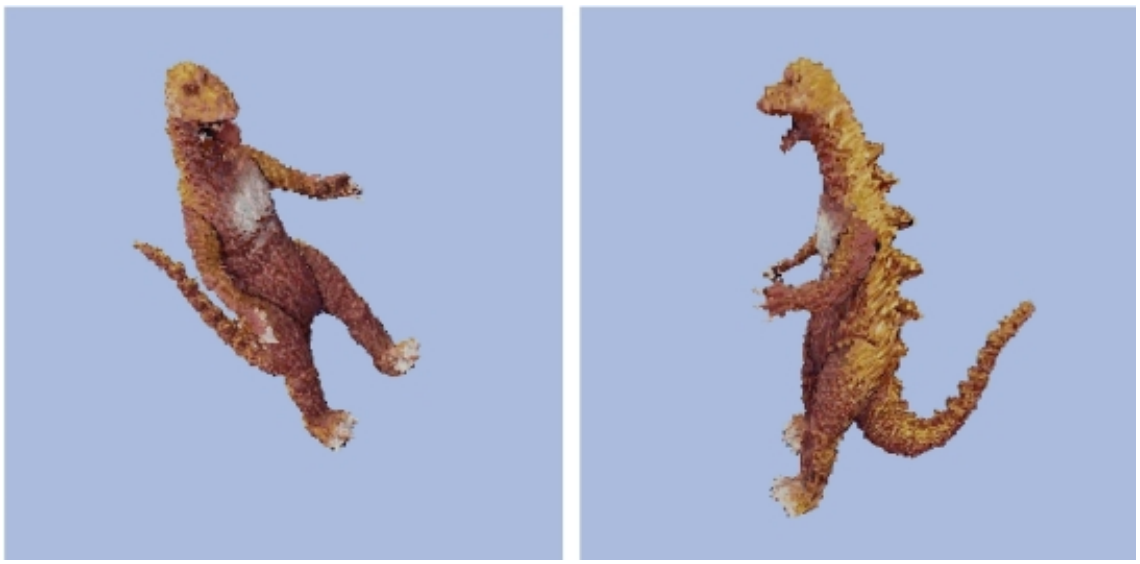

Fig. 12. Reconstructed dinosaur.

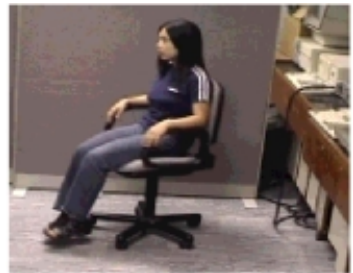

(a)

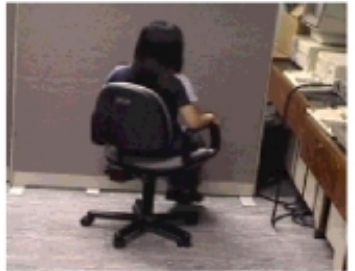

(b)

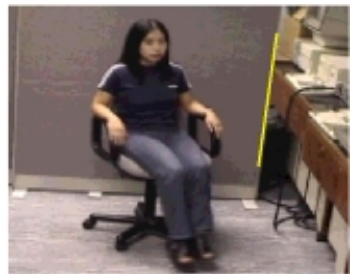

(c)

Fig. 13. Three images captured from the video. The video is a girl in a rotating chair. A vertical line in the background is marked out for searching the vanishing point along the rotation axis. 

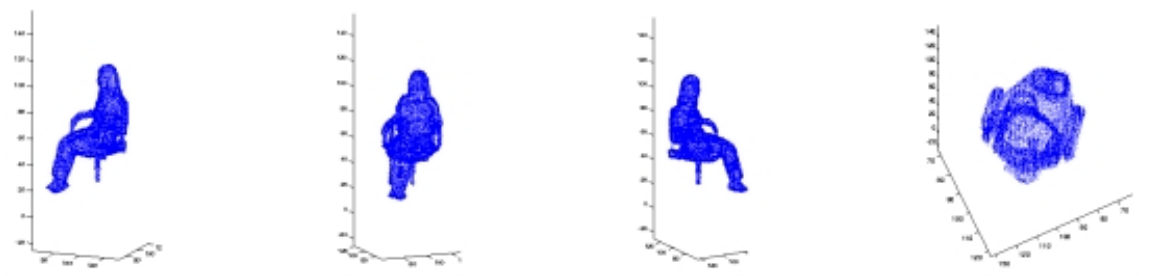

Fig. 14. 3D points of reconstructed girl from four different views.
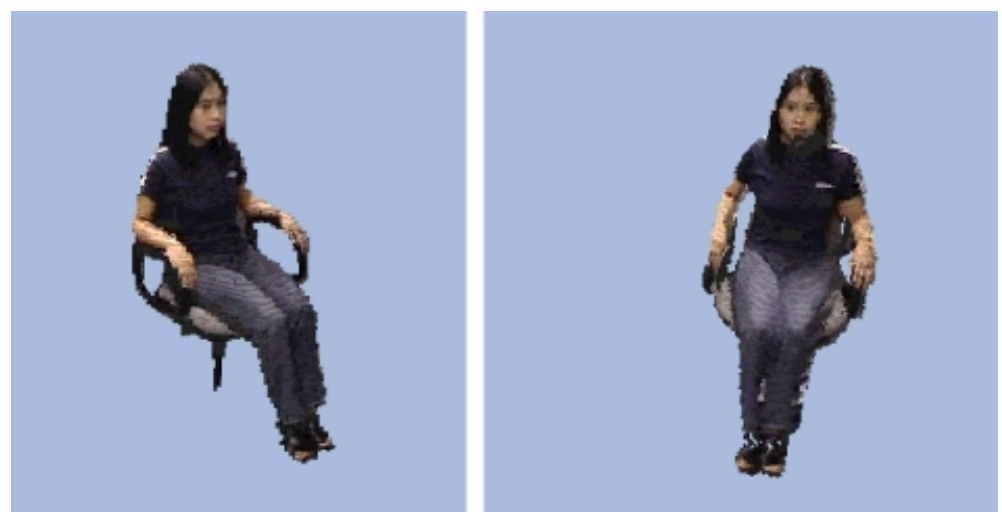

Fig. 15. Reconstructed girl.

Acknowledgments. This research is partially supported by RGC grant CUHK 4310/98E.

\section{References}

1. L. de Agapito, R. Hartley, and E. Hayman: Linear self-calibration of a rotating and zooming camera, CVPR, Vol. 1, pp. 15-21, 1999.

2. M. Armstrong, A. Zisserman, and R. Hartley: Self-Calibration from Image Triplets, ECCV, pp. 3-16, 1996.

3. F. Bookstein: Fitting conic sections to scattered data, CVGIP, vol. 9, pp. 56-71, 1979.

4. E. Boyer: Object models from contour sequences, ECCV, pp. 109-118, 1996.

5. C.H. Chien and J.K. Aggarwal: Identification of 3D objects from multiple silhouettes using quadtrees/octrees. Comp. Vision, Graphics, and Image Processing 36, 1986, pp.256-273.

6. O. Faugeras: Stratification of three-dimensional vision: projective, affine, and metric representation. J. Opt. Soc. Am., A, Vol. 12, pp. 465-484, 1995. 
7. A. Fitzgibbon, M. Pilu, and R.B. Fisher: Direct least square fitting of ellipses. PAMI, Vol. 21, No. 5, pp. 476-480, 1999.

8. A.W. Fitzgibbon, G. Cross, and A. Zisserman: Automatic 3D Model Construction for Turn-Table Sequences, SMILE Workshop, Freiburg, Germany, pp. 155-170, 1998.

9. R. Hartley: Euclidean reconstruction from uncalibrated views, In J. Mundy, A. Zisserman, and D. Forsyth, editors, Applications of Invariance in Computer Vision, LNCS 825, SpringerVerlag, pp. 237-256, 1994.

10. R. Hartley and A. Zisserman: Multiple View Geometry in Computer Vision, Cambridge University Press, 2000.

11. G. Jiang, H.T. Tsui, L. Quan and S.Q. Liu: Recovering the Geometry of Single Axis Motions by Conic Fitting, CVPR, pp. 293-298, 2001.

12. J.J. Koenderink and A.J. van Doorn: Affine structure from motion. J. Opt. Soc. Am., A, 8(2), pp. 377-385, 1991.

13. D. Liebowitz and A. Zisserman: Metric rectification for perspective images of planes. CVPR, pp. 482-488, 1998.

14. Q.-T. Luong and O.D. Faugeras: The Fundamental matrix: theory, algorithms, and stability analysis, IJCV, pp. 43-76, 1996.

15. P.R.S. Mendonça, K.-Y.K. Wong, and R. Cipolla: Camera pose estimation and reconstruction from image profiles under circular motion. In D. Vernon, editor, Proc. 6th European Conf. on Computer Vision, volume II, pp. 864-877, Dublin, Ireland, Jun 2000. Springer-Verlag.

16. J. Mundy, and A. Zisserman: Repeated Structures: Image Correspondence Constraints and Ambiguity of 3D Reconstruction, In Applications of invariance in computer vision, SpringerVerlag, 1994.

17. W.Niem: Robust and Fast Modelling of 3D Natural Objects from Multiple Views, SPIE, San Jose, Vol. 2182, pp. 388-397, 1994.

18. W. Niem, and R. Buschmann: Automatic Modelling of 3D Natural Objects from Multiple Views, Yakup Paker and Sylvia Wilbur: Image Processing for Broadcast and Video Production. Workshops in computing series, Springer, Hamburg, 1994.

19. H.S. Sawhney, J. Oliensis, and A.R. Hanson: Image Description and 3-D Reconstruction from Image Trajectories of Rotational Motion. PAMI, Vol. 15, No. 9, pp. 885-898, 1993.

20. J. Semple, and G. Kneebone: Algebraic Projective Geometry. Oxford University Press, 1952.

21. P.F. Sturm, and S.J. Maybank: On plane-based camera calibration: A general algorithm, singularities, applications, CVPR, pp. 432-437, 1999.

22. S. Sullivan, and J. Ponce: Automatic model construction, pose estimation, and object recognition from photographs using triangular splines, PAMI, Vol. 20, No. 10, pp. 1091-1097, 1998.

23. R. Szeliski: Shape from rotation, CVPR, pp. 625-630, 1991.

24. A. Zisserman, D. Liebowitz, and M. Armstrong: Resolving Ambiguities in AutoCalibration, In Philosophical Transactions of the Royal Society of London, SERIES A, vol. 356, no. 1740, pp. 1193-1211, 1998. 